\title{
A novel toolbox for $E$. coli lysis monitoring
}

\author{
Vignesh Rajamanickam $^{1,2}$ • David Wurm ${ }^{1}$ - Christoph Slouka ${ }^{1}$ Christoph Herwig ${ }^{1,2}$. \\ Oliver Spadiut ${ }^{1,2}$ (D)
}

Received: 21 June 2016 /Revised: 2 August 2016 / Accepted: 24 August 2016 / Published online: 2 September 2016

(C) The Author(s) 2016. This article is published with open access at Springerlink.com

\begin{abstract}
The bacterium Escherichia coli is a well-studied recombinant host organism with a plethora of applications in biotechnology. Highly valuable biopharmaceuticals, such as antibody fragments and growth factors, are currently being produced in E. coli. However, the high metabolic burden during recombinant protein production can lead to cell death, consequent lysis, and undesired product loss. Thus, fast and precise analyzers to monitor $E$. coli bioprocesses and to retrieve key process information, such as the optimal time point of harvest, are needed. However, such reliable monitoring tools are still scarce to date. In this study, we cultivated an E. coli strain producing a recombinant single-chain antibody fragment in the cytoplasm. In bioreactor cultivations, we purposely triggered cell lysis by $\mathrm{pH}$ ramps. We developed a novel toolbox using UV chromatograms as fingerprints and chemometric techniques to monitor these lysis events and used flow cytometry (FCM) as reference method to quantify viability offline. Summarizing, we were able to show that a novel
\end{abstract}

Published in the topical collection Process Analytics in Science and Industry with guest editor Rudolf W. Kessler.

Electronic supplementary material The online version of this article (doi:10.1007/s00216-016-9907-z) contains supplementary material, which is available to authorized users.

Oliver Spadiut

oliver.spadiut@tuwien.ac.at

1 Research Division Biochemical Engineering, Institute of Chemical Engineering, Vienna University of Technology, Getreidemarkt 9/166, 1060 Vienna, Austria

2 Christian Doppler Laboratory for Mechanistic and Physiological Methods for Improved Bioprocesses, Institute of Chemical Engineering, Vienna University of Technology, Gumpendorfer Strasse 1a, 1060 Vienna, Austria toolbox comprising HPLC chromatogram fingerprinting and data science tools allowed the identification of $E$. coli lysis in a fast and reliable manner. We are convinced that this toolbox will not only facilitate $E$. coli bioprocess monitoring but will also allow enhanced process control in the future.

Keywords Bioprocess monitoring · Data science tools . Lysis $\cdot$ Escherichia coli $\cdot$ Chromatogram fingerprinting · HPLC

\section{Introduction}

Escherichia coli is one of the most popular host organisms for recombinant protein production (e.g., [1, 2]). However, strong induction of recombinant protein production results in great cell stress and high metabolic burden, potentially leading to cell death and lysis [3]. Therefore, it is of utmost importance to monitor the physiological state of the cells to minimize product loss. Flow cytometry (FCM) is the predominant method to monitor and quantify E. coli cell death. However, FCM devices are expensive and therefore often not available. Furthermore, FCM measurements need manual intervention and often require time-consuming, offline sample preparation. In contrast, spectroscopic methods, such as RAMAN and near infrared spectroscopy (NIR), can be used for online monitoring $[4,5]$. Owing to the high magnitude of multi-dimensional data derived from these methods, multivariate data analysis (MVDA) is used for data interpretation [6, 7]. However, the continuously changing media background, changing morphologies, as well as changing process parameters (e.g., aeration) cause inaccuracy in measurements and thus limit the applications of these methods. Thus, alternative strategies for bioprocess monitoring are needed. 
In this study, we developed a novel toolbox based on UV chromatograms as fingerprints to identify $E$. coli cell lysis. To date, UV spectroscopy coupled to high pressure liquid chromatography (HPLC) is implemented for real-time monitoring in downstream processes [8]. However, we hypothesized that $\mathrm{UV}$ chromatographic data of $E$. coli bioprocess samples contain information about impurity release and lysis events and thus can also be used in upstream processing. We followed the impurity pattern of nucleic acids at $260 \mathrm{~nm}$ as marker for cell lysis along different $E$. coli bioprocesses. We combined UV chromatographic data with chemometric methods to identify lysis which may be used to define the optimal time point of harvest.

\section{Materials and methods}

\section{Strain}

E. coli BL21 (DE3) (Life technologies, CA, USA) and the pET28a $(+)$ expression vector were used for the production of the cytoplasmic recombinant single-chain antibody fragment (scFv).

\section{Bioreactor cultivations}

In all cultivations, a minimal medium according to DeLisa [9] supplemented with $0.02 \mathrm{~g} / \mathrm{L}$ Kanamycin was used. Three cultivations were carried out in a DASGIP multi bioreactor system with four glass bioreactors and a working volume of $2 \mathrm{~L}$ each (Eppendorf, Germany). Detailed information about this fermenter setup can be found elsewhere [10].

An overnight preculture was used for initiating the batch phase, followed by a fed-batch phase and a subsequent induction phase (addition of $0.1 \mathrm{mM} \mathrm{IPTG).} \mathrm{pO}_{2}$ and temperature were controlled throughout cultivation at $30 \%$ and $35{ }^{\circ} \mathrm{C}$, respectively. The $\mathrm{pH}$ during batch and non-induced fed-batch was kept constant at 7.2. During the induced fed-batch, the $\mathrm{pH}$ was either kept constant at 7.2 (Run1), or ramped from 7.2 to 5.7 (Run2) or from 7.2 to 8.7 (Run3) as shown in Electronic Supplementary Material (ESM) Table S1. Samples were taken every hour throughout the induction phase for offline determination of cell death by FCM and for chromatogram fingerprinting.

\section{Flow cytometry}

FCM was carried out according to Langemann et al. [11]. In short, cultivation broth was diluted to stay within the linear range of the detector of the FCM device (CyFlow ${ }^{\circledR}$ Cube 8 flow cytometer, Partec, Münster, Germany). After addition of the fluorescent dyes RH414 (abs./em. 532/760 nm, staining of all plasma membranes) and DiBAC4(3) (abs./em. 493/
$516 \mathrm{~nm}$, membrane potential-sensitive dye for assessment of viability), data were collected using the software CyView Cube 15 and analyzed with the software FCS Express V4 (DeNovo Software, Los Angeles, CA, USA). The error in FCM measurements was always below $5 \%$.

\section{Multivariate data analysis}

\section{Data acquisition}

A modular HPLC setup (PATfinder ${ }^{\mathrm{TM}}$ ) with an autosampler (Optimas), pump module (Azura P $6.1 \mathrm{~L}$ ), a multi-wavelength UV detector (Azura MWD 2.1 L) and a monolithic CIMac QA column $(0.1 \mathrm{~mL})$ was purchased from BIA separations (Ljubljana, Slovenia). Cell-free culture supernatants were diluted 1:5 with loading buffer (50 mM Tris-HCl, pH 8; AEX-A) to avoid deviations in the background matrix. Then, $50 \mu \mathrm{L}$ of the prepared samples were loaded onto the column and bound proteins and nucleic acids were eluted using a linear gradient with $50 \mathrm{mM}$ Tris-HCl $+1 \mathrm{M} \mathrm{NaCl}, \mathrm{pH} 8$ (AEX-B). Summarizing, column equilibration was done for 20 column volumes (CVs) with AEX-A, followed by sample injection, $10 \mathrm{CVs}$ post-injection wash with AEX-A and elution with a linear gradient of AEX-B for 20 CVs. The time required for acquiring chromatographic data of one sample was shorter than $5 \mathrm{~min}$. The column was cleaned with $1 \mathrm{M} \mathrm{NaOH}+2 \mathrm{M} \mathrm{NaCl}$ for $10 \mathrm{CV}$ after each sample to avoid carry-over. The flow velocity was kept constant at $283 \mathrm{~cm} / \mathrm{h}$. UV chromatographic data at $260 \mathrm{~nm}$ were recorded to follow release of nucleic acids. The chromatographic data were logged at a frequency of $5 \mathrm{~Hz}$.

\section{Data preprocessing}

UV chromatographic raw data are usually attributed with shifts along the retention time and the baseline, which both strongly influence further data analysis. In order to overcome these shortcomings, peak alignment and baseline correction were done using icoshift [12] and firstorder derivative, respectively. The preprocessed chromatographic UV data were then arranged as chromatogram fingerprints for further data analysis. Chromatogram fingerprints can be defined as a set of preprocessed overlaid chromatographic data which can be compared to identify and explain phenomena in a process. In our study, mean-centering and scaling of the UV chromatograms at $260 \mathrm{~nm}$ as fingerprints were done prior to performing PCA using SIMCA (Umetrics, Umea, Sweden). 


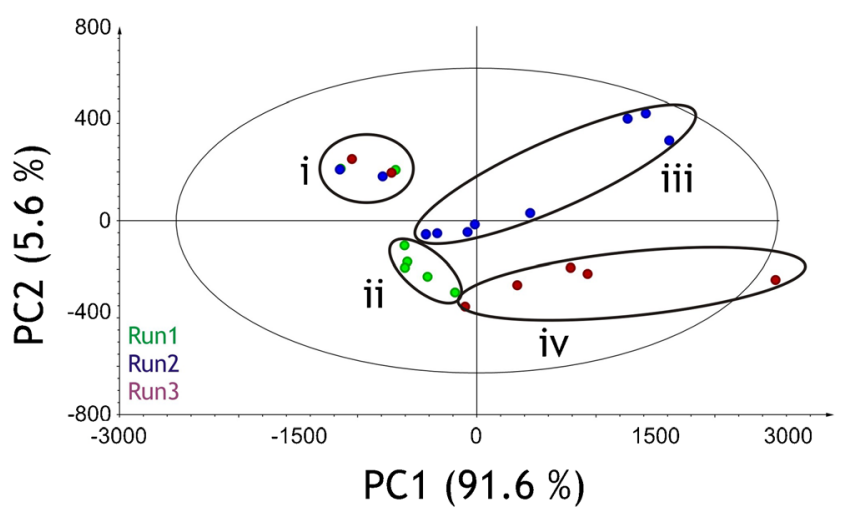

Fig. 1 PCA score plot depicting the variation in chromatogram fingerprints at $260 \mathrm{~nm}$ of the three different $E$. coli bioprocesses. Cluster $i$, samples prior to lysis trigger; cluster $i i$ with green circles, scores from Run1; cluster iii with blue circles, scores from Run2; and cluster iv with red circles, scores from Run3. Goodness of fit $\left(R^{2}\right)$ : 0.997; goodness of prediction $\left(Q^{2}\right): 0.994$

\section{Pattern recognition using $P C A$}

Principal component analysis is a widely used exploratory technique which helps in decomposing huge datasets such as the matrix $\mathrm{X}$ of the chromatogram fingerprints. The matrix $\mathrm{X}$ is represented after PCA by few latent variables, called principal components (PCs). The transformation of $\mathrm{X}$ to PCs results in different attributes that are associated with $\mathrm{X}$, called scores and loadings. The loadings of the PCs provide an overview of the variability in the $\mathrm{X}$ matrix. In general, the first PCs explain most of the variance in $\mathrm{X}$. The loadings explain at which retention time the variance in the chromatographic data was significant. For example, the first loading would show at which retention time the variance in the chromatographic data was high. An overview of scores plotted along different PCs reveals groupings/clusters explaining similar trends and/or deviations between different samples in $\mathrm{X}$.

Although the PCA score plots can be interpreted to monitor bioprocesses with respect to various PCs [13], multi-dimensional analysis of scores and loadings is cumbersome. Therefore, we used a univariate statistic (Hotelling's T2) from the PCA model to follow deviations from pre-defined operating conditions in the $E$. coli bioprocesses [13].

\section{Software}

Preprocessing of chromatographic data was done in MATLAB R2015a v8.5 (Mathworks, MA, USA). Pattern recognition using PCA was done in SIMCA v13.0 (Umetrics, Umea, Sweden).

\section{Results}

\section{Data acquisition and preprocessing}

UV chromatographic data were acquired using a UV-VIS detector at $260 \mathrm{~nm}$. After preprocessing, UV chromatographic data at $260 \mathrm{~nm}$ were arranged as chromatogram fingerprints as shown in ESM Fig. S1.
Fig. 2 FCM offline data and the Hotelling's T2 statistics for the three $E$. coli bioprocesses. a FCM data depicting cell death over process time; b Hotelling's T2 statistics from the PCA model developed with chromatogram fingerprints at $260 \mathrm{~nm}$ over process time. Blue diamonds, Run 1; orange circles, Run2; and,gray triangles, Run3. Dotted line, process deviation from control limit

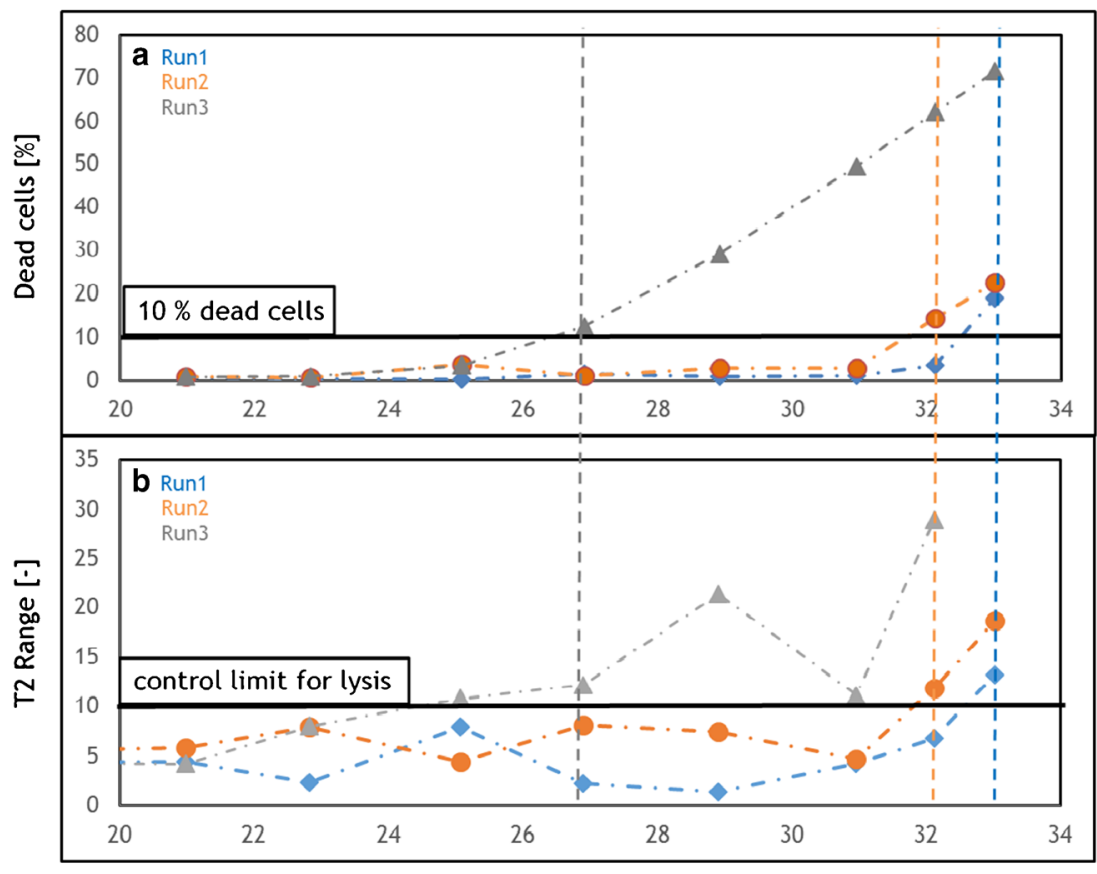

Process time [h] 


\section{Pattern recognition using $\mathrm{PCA}$}

A PCA model for UV chromatograms at $260 \mathrm{~nm}$ as fingerprints from the three different $E$. coli bioprocesses was established. We achieved a goodness of fit $\left(R^{2}\right)$ of 0.997 and goodness of prediction $\left(Q^{2}\right)$ of 0.994 with seven PCs from the PCA model. The PCA score plot for the UV chromatograms as fingerprints of the different experiments was used to identify clusters (similarities) and patterns (variances) along different PCs (Fig. 1). The significance level is indicated with an eclipse surrounding the scores as $95 \%$ confidence limit. It is interesting to note that the variance in chromatogram fingerprints during the initial phase of the different cultivations prior to intended $\mathrm{pH}$ ramps were similar. In other words, the impurity release pattern prior to $\mathrm{pH}$ ramps was similar in each bioprocess. From the score plots, we can speculate that after start of the $\mathrm{pH}$ ramps the impurity pattern in each experiment changed. This can be seen in the deviation from initial conditions in cluster $\mathrm{i}$ and other groupings where lysis was triggered with $\mathrm{pH}$ ramps (clusters ii, iii, and iv).

Hotelling's T2 statistic explains how well a model explains the variances in the data with respect to PCs [13, 14]. During initial stages of bioprocess development, the control limits for the univariate Hotelling's T2 statistics need to be defined. Control limits can be established based on the T2 statistic value of the samples at the beginning of the cultivation where the impurity release patterns are similar. We defined the control limits based on the T2 statistic of the initial phase of the process under optimal conditions and found the control limit in the T2 range of 10 (Fig. 2). Once control limits are established, process deviations can be monitored and unwanted loss in product quality or quantity can be avoided.

The FCM offline data and the Hotelling's T2 statistics, calculated from the PCA model, are shown in Fig. 2. The Hotelling's T2 statistics showed clear deviations from the control limit in each bioprocess. In fact, these deviations happened at the same time when cell death increased (indicated by thin dotted lines in Fig. 2). Apparently, cells started to die at different time points due to the $\mathrm{pH}$ ramps. Cell death resulted in lysis and thus in the release of impurities (nucleic acids), which we were able to reliably detect by UV chromatograms as fingerprints and combined data analysis. Based thereon, the time point at which the bioprocess started to deviate from normal operating condition was defined as the optimal time point of harvest. With the implementation of this novel monitoring toolbox, online detection of physiological events in the bioreactor is possible, and cumbersome offline analytics along bioprocesses is minimized.

\section{Discussion}

We implemented a novel toolbox comprising UV chromatogram as fingerprints and chemometric techniques to monitor cell death in $E$. coli bioprocesses and to define the optimal time point of harvest.

The novelty of this approach is the use of whole UV chromatogram as fingerprints, rather than single chromatogram peaks, in combination with multivariate data analysis (MVDA) tools for monitoring of bioprocesses. Chromatogram fingerprinting approaches have been only used in chemical formulations and in some downstream bioprocesses so far, but for the first time we showed the applicability of this technique in upstream process monitoring. We envision the implementation of this toolbox for monitoring different unit operations in a bioprocess, such as bioreactor cultivations, harvesting, and product purification, which will facilitate continuous bioprocessing and process development.

Acknowledgments Open access funding provided by TU Wien (TUW). The authors would like to thank BIA separations (Slovenia) for providing the columns and technical support.

Authors' contributions David Wurm and Vignesh Rajamanickam designed the study. David Wurm, Christoph Slouka, and Vignesh Rajamanickam conducted the experiments and analyzed the data. Vignesh Rajamanickam developed the model. David Wurm, Oliver Spadiut, and Vignesh Rajamanickam wrote the paper. Christoph Herwig and Oliver Spadiut supervised the work.

\section{Compliance with ethical standards}

Conflict of interest The authors declare that they have no conflict of interest.

Open Access This article is distributed under the terms of the Creative Commons Attribution 4.0 International License (http:// creativecommons.org/licenses/by/4.0/), which permits unrestricted use, distribution, and reproduction in any medium, provided you give appropriate credit to the original author(s) and the source, provide a link to the Creative Commons license, and indicate if changes were made.

\section{References}

1. Rosano GL, Ceccarelli EA. Recombinant protein expression in Escherichia coli: advances and challenges. Front Microbiol. 2014;5:172. doi:10.3389/fmicb.2014.00172.

2. Sugiki T, Fujiwara T, Kojima C. Latest approaches for efficient protein production in drug discovery. Expert Opin Drug Discovery. 2014;9:1189-204. doi:10.1517/17460441.2014.941801.

3. Marschall L, Sagmeister P, Herwig C. Tunable recombinant protein expression in E. coli: enabler for continuous processing? Appl Microbiol Biotechnol. 2016;100:5719-28. doi:10.1007/s00253016-7550-4.

4. Chiang Y-H, Wu SH, Kuo Y-C, et al. Raman spectroscopy for grading of live osteosarcoma cells. Stem Cell Res Ther. 2015;6: 81. doi:10.1186/s13287-015-0074-5. 
5. Rantanen J, Wikstrom H, Turner R, Taylor LS. Use of in-line nearinfrared spectroscopy in combination with chemometrics for improved understanding of pharmaceutical processes. Anal Chem. 2005;77:556-63. doi:10.1021/ac048842u.

6. Read EK, Park JT, Shah RB, et al. Process Analytical Technology (PAT) for biopharmaceutical products: part I. Concepts and applications. Biotechnol Bioeng. 2010;105: 276-84. doi:10.1002/bit.22528.

7. Rathore AS, Parr L, Dermawan S, et al. Large scale demonstration of a process analytical technology application in bioprocessing: use of on-line high performance liquid chromatography for making real time pooling decisions for process chromatography. Biotechnol Prog. 2010;26:448-57. doi:10.1002/btpr.320.

8. Rathore AS, Yu M, Yeboah S, Sharma A. Case study and application of process analytical technology (PAT) towards bioprocessing: use of on-line high-performance liquid chromatography (HPLC) for making real-time pooling decisions for process chromatography. Biotechnol Bioeng. 2008;100:306-16. doi:10.1002/bit.21759.

9. DeLisa MP, Li J, Rao G, et al. Monitoring GFP-operon fusion protein expression during high cell density cultivation of
Escherichia coli using an on-line optical sensor. Biotechnol Bioeng. 1999;65:54-64.

10. Sagmeister P, Schimek C, Meitz A, et al. Tunable recombinant protein expression with E. coli in a mixed-feed environment. Appl Microbiol Biotechnol. 2014;98:2937-45. doi:10.1007/s00253-013-5445-1.

11. Langemann T, Mayr UB, Meitz A, et al. Multi-parameter flow cytometry as a process analytical technology (PAT) approach for the assessment of bacterial ghost production. Appl Microbiol Biotechnol. 2016;100:409-18. doi:10.1007/s00253015-7089-9.

12. Tomasi G, Savorani F, Engelsen SB. icoshift: an effective tool for the alignment of chromatographic data. J Chromatogr A. 2011;1218:7832-40. doi:10.1016/j.chroma.2011.08.086.

13. Nucci ER, Cruz AJG, Giordano RC. Monitoring bioreactors using principal component analysis: production of penicillin $\mathrm{G}$ acylase as a case study. Bioprocess Biosyst Eng. 2010;33:557-64. doi:10.1007/s00449-009-0377-y.

14. Eriksson L, Kettaneh-Wold N, Johansson E, et al. Multi- and megavariate data analysis. Umea: MKS Umetrics AB; 2006. 